\title{
Nonconvulsive Epileptic Seizure Detection in Scalp EEG Using Multiway Data Analysis
}

\author{
Yissel Rodríguez Aldana, \\ Borbála Hunyadi, Member, IEEE, \\ Enrique J. Marañón Reyes, \\ Valia Rodríguez Rodríguez, \\ and Sabine Van Huffel, Fellow, IEEE
}

\begin{abstract}
Nonconvulsive status epilepticus is a condition where the patient is exposed to abnormally prolonged epileptic seizures without evident physical symptoms. Since these continuous seizures may cause permanent brain damage, it constitutes a medical emergency. This paper proposes a method to detect nonconvulsive seizures for a further nonconvulsive status epilepticus diagnosis. To differentiate between the normal and seizure electroencephalogram (EEG), a K-Nearest Neighbor, a Radial Basis Support Vector Machine, and a Linear Discriminant Analysis classifier are used. The classifier features are obtained from the Canonical Polyadic Decomposition (CPD) and Block Term Decomposition (BTD) of the EEG data represented as third order tensor. To expand the EEG into a tensor, Wavelet or Hilbert-Huang transform are used. The algorithm is tested on a scalp EEG database of 139 seizures of different duration. The experimental results suggest that a Hilbert-Huang tensor representation and the CPD analysis provide the most suitable framework for nonconvulsive seizure detection. The Radial Basis Support Vector Machine classifier shows the best performance with sensitivity, specificity, and accuracy values over $98 \%$. A rough comparison with other methods proposed in the literature shows the superior performance of the proposed method for nonconvulsive epileptic seizure detection.
\end{abstract}

Index Terms-Hilbert Huang Transform, Multiway Data Analysis, Nonconvulsive epileptic seizures, Wavelet Transform.

\section{INTRODUCTION}

This work has been supported by the Belgian foreign Affairs-Development Cooperation through VLIR-UOS (2013-2019) (Flemish Interuniversity Council-University Cooperation for Development) in the context of the Institutional University Cooperation program with Universidad de Oriente.

The research leading to these results has received funding from imec funds 2017 and the European Research Council under the European Union's Seventh Framework Programme (FP7/2007-2013) / ERC Advanced Grant: BIOTENSORS (no. 339804). This paper reflects only the authors' views and the Union is not liable for any use that may be made of the contained information.

Y. Rodríguez Aldana is with the Universidad de Oriente. Center of Neuroscience and Signals and Image Processing, Santiago de Cuba, Cuba.(email:yadana@uo.edu.cu)

B. Hunyadi is with Stadius Center for Dynamical Systems, Signal Processing and Data Analytics and IMEC, Leuven, BE. Leuven, Belgium.(email:borbala.hunyadi@esat.kuleuven.be)

E.J. Marañón Reyes is with the Universidad de Oriente. Center of Neuroscience and Signals and Image Processing, Santiago de Cuba, Cuba.(email:emaraon@uo.edu.cu)

V. Rodríguez Rodríguez is with ICU-Almeijeiras Clinic Surgical Hospital, La Habana, Cuba and Aston University, United Kingdom. (e-mail: v.rodriguez@aston.ac.uk)

S. Van Huffel is with Stadius Center for Dynamical Systems, Signal Processing and Data Analytics and IMEC, Leuven, BE. Leuven, Belgium.(email:sabine.vanhuffel@esat.kuleuven.be)
Q TATUS epilepticus (SE) is a condition where the patient $\checkmark$ is exposed to abnormally prolonged epileptic seizures. This condition could have long-term consequences as neuronal death, neuronal injury, and alteration of neuronal networks, depending on the seizure type and duration [1]. SE can be divided into two major categories, convulsive SE (CSE) and nonconvulsive SE (NCSE). CSE is characterized by muscular spasms typical for epileptic convulsive seizures (ECS). On the other hand, NCSE symptoms are too subtle to be noticed since this status is characterized by nonconvulsive seizures, which show persistent epileptic activity in the EEG without evident physical symptoms.

Nonconvulsive epileptic seizures (NCES) can be found associated with coma/stupor or not (i.e. absence status, focal SE). Patients without coma/stupor are often awake and are treated in emergency services. Symptoms such as automatisms, facial twitching, eye deviation or jerking might help NCES diagnosis. On the other hand, patients with coma/stupor treated at Intensive Care Units (ICU) are usually unconscious or unresponsive. Consequently, these NCES have no physical observable symptoms at all. Then, the diagnosis can only be accomplished by means of EEG monitoring. As explained before, these seizures are often associated with serious brain damage with very poor prognosis [2]. Therefore, it is crucial to monitor the EEG at the ICU, in order to allow physicians to detect a seizure when it is still possible to prevent it from causing permanent brain damage.

Due to the nature of long-term EEG recordings, its analysis is a time-consuming task. This analysis can be performed by doctors or seizure detection software. The visual analysis performed by clinicians might lead to intra-observational inconsistencies in the results (the same expert can produce different outputs if (s)he reviews the data at different times) [3]. Software approaches are more time efficient, more consistent and objective.

Most of the available automated seizure detection software has been extensively trained on seizures obtained from patients with seizure disorders without brain injuries. Nevertheless, these seizures and those from comatose/brain-injured patients are different. The ictal activity from brain-injured/comatose patients has a longer duration, is less defined in time (unclear on-set /off-set), less organized and lower in maximum frequency [3].

A big number of published seizure detection methods ad- 
dress CES [4], [5] and only a few of them address NCES [6]. The proposed methods for NCES detection published until 2015 are summarized in the work of Ansari and Sharma [6]. These methods can be split according to their principal aim: those that detect NCES without coma/stupor (i.e. absence seizures) [7], [8], [9], [10], [11] and the ones that perform the NCES detection on ICU patients [12], [13], [14], [15], [16]. All databases used are private and very dissimilar in size and composition, thereby complicating objective comparison between the diverse methods.

The methodology used in these studies is also very diverse. Classifiers such as Support Vector Machines, Neural Networks, and Linear Discriminant functions are the most commonly used ones. For the data description, the most widespread features are Wavelet Transform (WT) scales [7], [8], [9], [16], Entropy [10], [11] and nonlinear parameters [8], [11], [12]. The major drawbacks of these methods are related to the arbitrary nature of the selected thresholds for performing the detection. Seizure duration and the number of channels affected by the seizure activity are the most popular criteria for thresholding [9], [14], [15], [16]. Seizures are not detected if they are too short in time or too localized (just a few channels affected by the seizure activity). NCES are heterogeneous across patients, the patterns present in the EEG will depend on the etiology of the seizure. Therefore a threshold which works for one patient will not necessarily work for another. Also, there are more meaningful ways to describe the seizure's spatial localization than the number of channels; that is, the seizure topography.

This research aims to propose a method to detect NCES, which alleviates these shortcomings. In particular, this method attempts to identify the NCES based on their similarity to the first NCES detected by the physician at the EEG [17]. The explored features are obtained by means of a multiway analysis of the EEG signal represented as a third order tensor $X \in \mathbb{R}^{(F \times T \times C h)}$ with axes frequency $\times$ time $\times$ channels. The tensors are computed by expanding the EEG segments using WT or Hilbert Huang Transform (HHT). WT is a popular tool for seizure EEG tensor construction [18], [19]. However, we propose the HHT since it resolves time $\times$ frequency events with a finer resolution than WT and thanks to the adaptive nature of the Empirical Mode Decomposition (EMD) provides a more meaningful physical interpretation of the underlying EEG data. The tensor decomposition is performed with CPD and BTD. It has been shown that tensor decompositions of multiway EEG representations can extract seizure sources and accurately characterize the seizure pattern [18], [19], [20]. The multiway analysis exploits the EEG high dimensional structure by analyzing its spectral, temporal and spatial properties simultaneously. As such, this approach represents a neat and practical alternative to analyze large, heterogeneous and multidimensional feature sets. Moreover, it eliminates the need for thresholding since the classifiers will implicitly evaluate the similarity of the signatures. Fig.1 displays the general block diagram of the proposed method. Clinically, the goal of the algorithm is twofold. First, it indicates all seizure occurrences (sz flag). Besides, it sets an alarm at the very first seizure, indicating that the patient has a seizure disorder, and subsequently set an alarm in case of continuous seizure activity, indicating that the patient might develop status epilepticus.

\section{Material AND Methods}

\section{A. EEG Data}

EEG data were collected as part of patients' clinical assessment at the Epilepsy Unit of the Cuban Neurological Restoration Center (CIREN) and at the ICU of the Clinical Surgical Hospital Hermanos Ameijeiras, both in Havana City. Data were anonymized prior to its use in this study. The visual inspection and labeling were performed by three neurophysiologists (including VRR). Since the patients come from different hospitals and areas, the acquisition protocol shows some differences; yet the sampling rate for all recordings is $200 \mathrm{~Hz}$. The number of electrodes used for the recordings varies between 8 and 19. In all cases, the electrodes were placed according to the 10-20 montage system. The use of the 10-20 montage for all records guarantees that the electrodes' name and position do not change in the records.

The dataset comprises 14 adult patients between 18 and 57 years old with various brain disorders that present NCES. A total of 139 seizures have been registered, all of them NCES according to the neurophysiologist' diagnosis (55 with coma/stupor). A more detailed description is given in Table I. All procedures were reviewed and approved by the Ethical Committees of the CIREN and Hermanos Ameijeiras Hospital respectively.

\section{B. Tensor formulation}

The EEG from each recording is divided into segments (epochs) of 3 seconds long. All epochs are expanded in the time-frequency domain using a WT or HHT. A $3^{\text {rd }}$ order tensor is built from every epoch with frequency $\times$ time $\times$ channels axes.

1) Wavelet Transform: The Continous WT (CWT) performs a multi-resolution analysis of a signal $\mathrm{x}(\mathrm{t})$ decomposing it into sub-band signals representing activity at different time scales. WT has been reported in the literature as one of the preferred techniques by the authors to perform the epileptic seizure detection [5]. The signal decomposition is achieved by convolving scaled and shifted versions of a mother wavelet $\psi(n)$ with $x(t)$ and it is defined as follows,

$$
x_{w}(a, b)=\frac{1}{\sqrt{|a|}} \int_{-\infty}^{\infty} x(t) \psi^{*}\left(\frac{t-b}{a}\right) d t
$$

Where ()$*$ indicates the complex conjugate, $\psi$ is the analyzing wavelet, a $(>0)$ is the scale parameter and b is the shift parameter [21].

To expand the EEG into a three-way tensor expressed as $X \in \mathbb{R}^{(F \times T \times C h)}$ the CWT is applied to each channel transforming it in a matrix, where the rows are the wavelet scales $(F)$, the columns time instants $(T)$ and the elements are coefficients. The $C h$ dimension represents the spatial distribution among the channels. Since the NCES activity is below $5 \mathrm{~Hz}$ [22], five wavelet scales were computed, which 


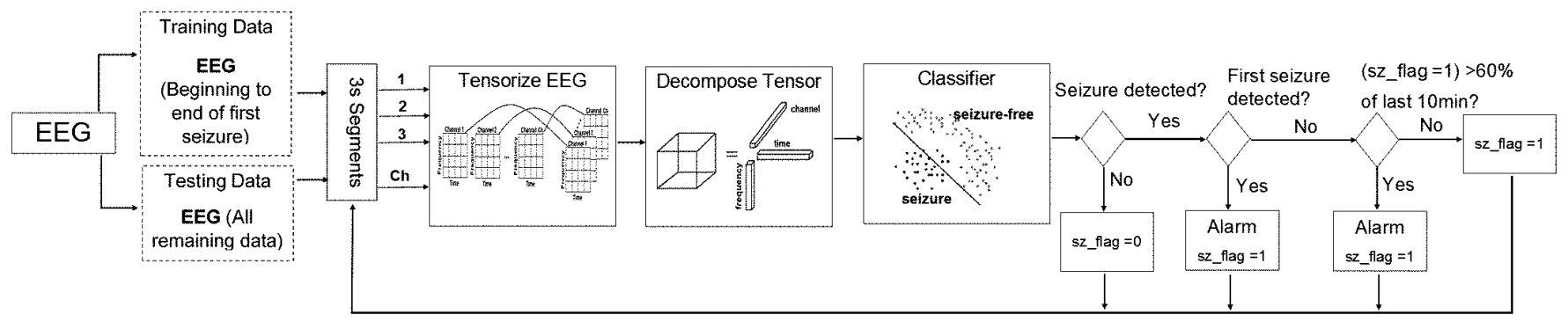

Fig. 1. General block diagram of the proposed method.

TABLE I

Detailed Description of THE EEG Database

\begin{tabular}{|c|c|c|c|c|c|c|c|c|c|c|}
\hline \multicolumn{4}{|c|}{ Patient data } & \multicolumn{2}{|c|}{ Record } & \multicolumn{5}{|c|}{ Protocol } \\
\hline Patient & Gender & Diagnosis & Type & Duration(h:min:s) & Seizures \# & Channels & $E K G$ & $E O G$ & $E M G$ & Video \\
\hline 1 & $M$ & Temporal lobe epilepsy. & Ambulatory & $00: 38: 48$ & 6 & 19 & $o$ & $o$ & $o$ & $o$ \\
\hline 2 & $M$ & Focal Epilepsy. & Ambulatory & $00: 16: 44$ & 3 & 19 & $o$ & $o$ & $o$ & $o$ \\
\hline 3 & $M$ & Temporal lobe epilepsy. & Ambulatory & $00: 48: 29$ & 13 & 19 & $o$ & $o$ & $o$ & $o$ \\
\hline 4 & $F$ & Partial Temporal Lobe Epilepsy. & Ambulatory & $00: 14: 05$ & 5 & 19 & $o$ & $o$ & $o$ & $o$ \\
\hline 5 & $F$ & Partial psychic seizures. & Ambulatory & $00: 16: 05$ & 2 & 19 & $o$ & $o$ & $o$ & $o$ \\
\hline 6 & $F$ & Lenns-Gastaut. Atonic Neck. & Ambulatory & $00: 39: 30$ & 2 & 19 & $o$ & $o$ & $o$ & $o$ \\
\hline 7 & $F$ & Head trauma. & Ambulatory & $00: 28: 15$ & 12 & 17 & $x$ & $x$ & $x$ & $o$ \\
\hline 8 & $F$ & Spike wave and Polispike wave. & Ambulatory & $08: 05: 58$ & 6 & 17 & $x$ & $x$ & $x$ & $o$ \\
\hline 9 & $M$ & Absence seizures. & Ambulatory & $07: 42: 52$ & 34 & 17 & $x$ & $x$ & $x$ & $o$ \\
\hline 10 & $F$ & Leukosytosis. & $I C U$ & $19: 36: 38$ & 40 & 19 & $x$ & $x$ & $x$ & $x$ \\
\hline 11 & $M$ & Higly malignant astrocytoma. & $I C U$ & $03: 34: 50$ & 2 & 13 & $x$ & $o$ & $x$ & $x$ \\
\hline 12 & $F$ & Unknown & $I C U$ & $21: 12: 46$ & 6 & 8 & $x$ & $x$ & $x$ & $x$ \\
\hline 13 & $F$ & Connective tissue mixed disease. & $I C U$ & $00: 47: 20$ & 5 & 19 & $x$ & $x$ & $x$ & $x$ \\
\hline 14 & $F$ & Cerebral gliomatosis. & $I C U$ & $01: 02: 32$ & 3 & 15 & $x$ & $x$ & $o$ & $x$ \\
\hline
\end{tabular}

correspond to $1-5 \mathrm{~Hz}$ frequencies, with $1 \mathrm{~Hz}$ resolution. As mother wavelet, a Mexican Hat is used as reported in [18], [19]. The tensors obtained with CWT will be referred further on as W_Tensor.

2) Hilbert-Huang Transform: The HHT is a method that uses two steps to analyze the data. First, the data are decomposed into a number of components and a residual using the EMD. Second, the Hilbert transform is applied to those components to construct a frequency $\times$ time $\times$ energy distribution, designated as the Hilbert spectrum. In this spectrum, all events in the time domain will be preserved by the instantaneous frequency computed by the Hilbert transform [23].

The main characteristic of EMD is to decompose a signal into a so-called Intrinsic Mode Function (IMF) plus a residue which is conventionally defined as the temporal trend of the series. By definition, an IMF satisfies two conditions: (i) for the entire data set, the number of extrema and the number of zero crossings must be either equal or differ at most by one and (ii) the mean at any point of the contour defined by interpolating the local maxima (upper envelope) and the contour defined by interpolating the local minima (lower envelope) is zero [24]. For any one-dimensional discrete signal, EMD can be presented with the following equation,

$$
x(t)=\sum_{k=1}^{n} i m f_{k}(t)+r(t)
$$

where $i m f_{k}$ is the $k^{t h}$ IMF of the signal, and $r$ is the residue [23].

Once the IMFs are computed each of them is transformed with the Hilbert Transform defined as,

$$
y_{i m f_{k}}(t)=\frac{1}{\pi} P \int_{-\infty}^{\infty} \frac{i m f_{k}(\tau)}{t-t_{0}} d t
$$

where $P$ indicates the Cauchy principal value. $y_{i m f_{k}}(t)$ and $i m f_{k}(t)$ form a complex conjugate pair that describes an analytic signal $z_{i m f_{k}}(t)$,

$$
z_{i m f_{k}}(t)=i m f_{k}(t)+j y_{i m f_{k}}(t)=a_{i m f_{k}}(t) e^{j \theta_{i m f_{k}}(t)}
$$

where $a_{i m f_{k}}$ is the instantaneous amplitude and $\theta_{i m f_{k}}$ is the instantaneous phase defined as,

$$
a_{i m f_{k}}(t)=\sqrt{\left[i m f_{k}(t)\right]^{2}+\left[y_{i m f_{k}}(t)\right]^{2}}
$$

and

$$
\theta_{i m f_{k}}(t)=\arctan \left(\frac{y_{i m f_{k}}(t)}{i m f_{k}(t)}\right)
$$

respectively.

Then the instantaneous frequency is computed as

$$
w_{i m f_{k}}(t)=\frac{d \theta_{i m f_{k}}(t)}{d t}
$$


The $x_{H H}(w, t)$ matrices are now constructed, representing the energy at each time point $t$ and at each instantaneous frequency $w$ the in following way,

$$
x_{H H}(w, t)=\sum_{\substack{k=1 \\ w=w_{i m f_{k}}(t)}}^{n} s_{i m f_{k}}(t)
$$

where the $\mathrm{n}$ is the number of IMFs and the energy $s_{i m f_{k}}$ is defined as

$$
s_{i m f_{k}}(t)=a_{i m f_{k}}(t)^{2}
$$

As implemented with CWT, the HHT is applied to every EEG channel. The Hilbert spectrum is truncated at $5 \mathrm{~Hz}$. The $x_{H H}(w, t)$ matrices are arranged as frontal slices of a tensor $X \in \mathbb{R}^{(F \times T \times C h)}$, further on defined as HH_Tensor, where $\mathrm{F}$ indicates the $1-5 \mathrm{~Hz}$ frequency values, $T$ the number of time samples and $C h$ the number of EEG channels.

\section{Tensor Decomposition}

The tensor obtained by WT or HHT will be analyzed using CPD and BTD. CPD provides a trilinear description of the data, which is a very compact and interpretable model. This model assumes that the seizure pattern maintains the same frequency and topography within a certain period of time. CPD has been applied successfully in several studies that represent the EEG as a third-order tensor to recognize epileptic seizure activity [18], [19], [20], [25]. This fact makes CPD an attractive method for our study. On the other hand, BTD is more flexible, and facilitates a more accurate model when the data frequency or topography change over time. Some studies have demonstrated that BTD outperforms CPD for EEG applications where the pattern of interest shows evolution in frequency, morphology and/or topography [19], [26]. Epileptic seizures have been reported as having such kind of behavior. Therefore, BTD exploration is also interesting for this application.

1) Canonical Polyadic Decomposition (CPD): The CPD represents a third-order tensor $X \in \mathbb{R}^{(F \times T \times C h)}$ as the outer product (represented as ' $\circ$ ') of rank-1 tensors in the following way,

$$
X \approx \sum_{r=1}^{R} a_{r} \circ b_{r} \circ c_{r}
$$

Where $a_{r} \in \mathbb{R}^{F}, b_{r} \in \mathbb{R}^{T}$, and $c_{r} \in \mathbb{R}^{C h}$ are nonzero vectors that define the mode-n signature, $n=1, \ldots, N$ (for $N=3$ ). With $1 \leq r \leq R$.

The tensor rank of $X$ is equal to the minimal number of components $\mathrm{R}$ that generates an 'exact' $\mathrm{CPD}$ of $X$, where 'exact' means that there is equality at (10) [27]. A tensor CPD is unique under mild conditions up to permutation and scaling [27]. Let $A=\left[a_{1} \ldots a_{R}\right], B=\left[b_{1} \ldots b_{R}\right]$ and $C=\left[c_{1} \ldots c_{R}\right]$ be the factor matrices corresponding to each mode- $n$, and $k_{A}$, $k_{B}$, and $k_{C}$ their respective $k$-rank. The $k$-rank $k_{A}$ of a matrix $A$ is the maximum value ensuring that any subset of $k_{A}$ columns are linearly independent, with $k_{A}<r_{A}\left(r_{A}\right.$ rank of the factor mattrix $A$ ). According to Kruskal's theorem [28] a sufficient condition for the uniqueness of a third-order tensor CPD up to permutation and scaling uncertainty is given by,

$$
k_{A}+k_{B}+k_{C} \geq 2 R+2
$$

2) Block Term Decomposition (BTD): The (L,M,O)-BTD of a third-order tensor $X \in \mathbb{R}^{(F \times T \times C h)}$ is written as,

$$
X=\sum_{r=1}^{R} D_{r} \bullet A_{r} A_{2} B_{r} \bullet_{3} C_{r}
$$

where $\bullet_{n}$ denote the mode-n product, $D_{r} \in \mathbb{R}^{(L \times M \times O)}$ are full rank - $(L, M, O), A_{r} \in \mathbb{R}^{(F \times L)}$ (with $F \geq L$ ), $B_{r} \in \mathbb{R}^{(T \times M)}$ (with $T \geq M$ ), and $C_{r} \in \mathbb{R}^{(C h \times O)}$ (with $C h \geq O$ ) are full column rank, $1 \leq r \leq R$. BTD is unique up to permutation and scaling inherited from CPD under more restrictive conditions. Essential uniqueness conditions for the (L,M,O)-BTD can be found in [29].

The two tensor sets obtained, W_Tensor and HH_Tensor, are decomposed using, CPD and BTD. The EEG epochs that contain seizure activity will be labeled as positives and the rest as negatives.

To differentiate the features sets, those obtained from a tensor built with HHT are denoted by ' $h$ ', ' $w$ ' is used for those obtained with WT. Space, Frequency and Time signatures obtained from the tensor decomposition, are referred as 'spac', 'freq', and 'time'. The training using all assembled signatures together is specified as 'asm'. To tag the decomposition method 'CP' and 'BT'will be used to refer to CPD and BTD respectively. As an example, the Space signature obtained by the CPD decomposition of a tensor built with WT, will be denoted as: w_spac_CP.

\section{Multilinear Rank Analysis}

Before performing CPD and BTD, the tensor rank must be determined. The multilinear rank ( $\left.m l \_r a n k\right)$ of a high-order tensor is the $n$-tuple of the mode- $n$ ranks. Contrary to the matrix case, each mode- $n$ rank may be different. In order to find the best low $m l$ rank approximation for the computed tensors, the multilinear singular value decomposition (MLSVD) is used [30]. The MLSVD is a multilinear generalization of the matrix SVD. The MLSVD of a third-order tensor $X \in \mathbb{R}^{(F \times T \times C h)}$ can be written as,

$$
X=Z \bullet_{1} U^{(1)} \bullet_{2} U^{(2)} \bullet_{3} U^{(3)}
$$

where $U^{(n)}, n=1, \ldots, N, N=3$, are the orthonormal basis for the $n$ different subspaces of the mode- $n$ vectors and $Z \in \mathbb{R}^{(L \times M \times O)}$ is an all-orthogonal and ordered tensor [31]. Essentially, the MLSVD considers a given tensor as $\mathrm{N}$ sets of mode- $n$ vectors and computes the matrix SVD of these sets. The truncation of the MLSVD gives a suboptimal solution to the tensor best low $m l$ rank approximation problem which can be used as initialization to iterative algorithms such as the low multilinear rank approximation (LMLRA). The LMLRA can be formulated as MLSVD, the difference between these two algorithms lies in the computation and the optimality of the decomposition. LMLRA refines the MLSVD initial guess using an optimization based method (i.e. nonlinear 

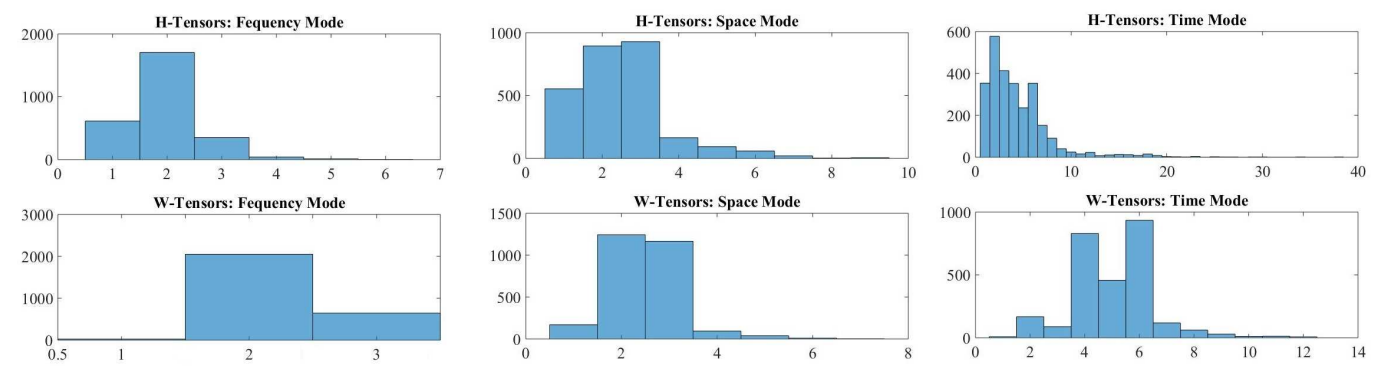

Fig. 2. LMLRA analysis Frequency, Time and Space modes histograms. Axis X and Y represent the number of components and tensors respectively.
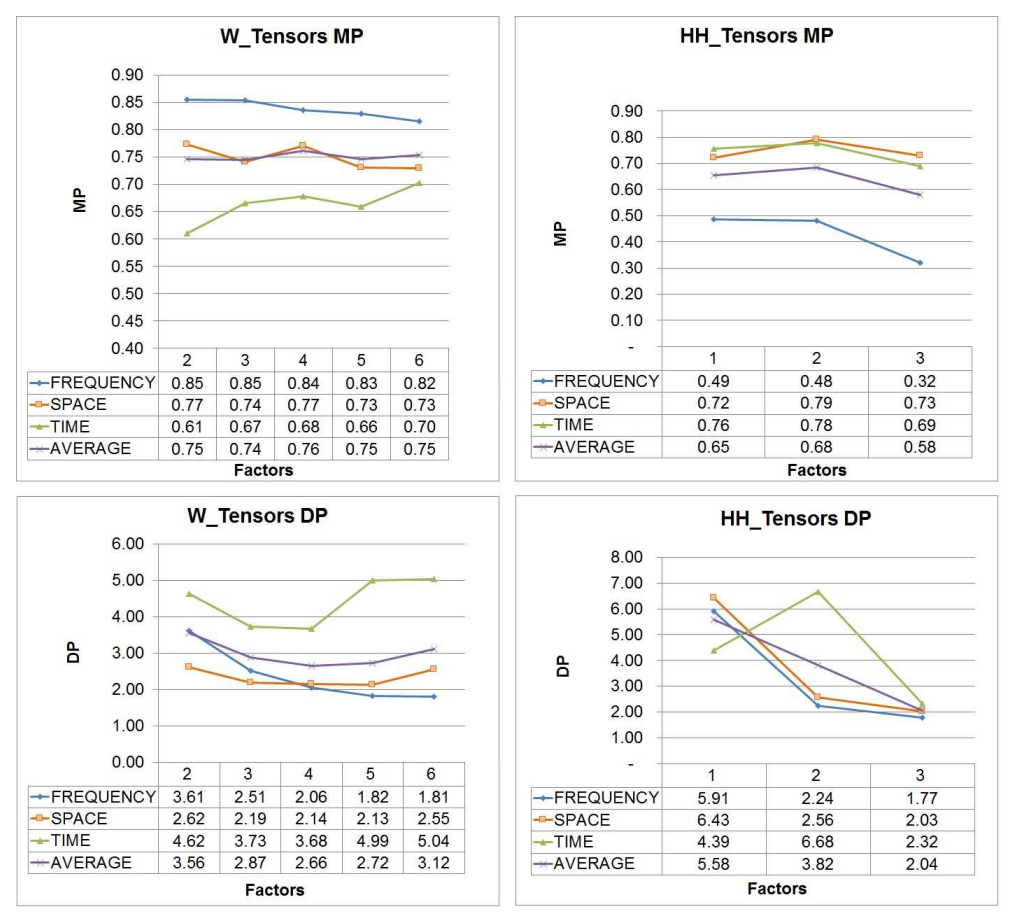

Fig. 3. Obtained MP and DP for HH_Tensors and W_Tensor CPD signatures computed for several numbers of factors. The displayed MP values are computed for the features matrices of the first $\bar{N}$ CES. The MP and DP values displayed on the charts are averaged over the number of features of each mode.

unconstrained optimization, nonlinear least squares, adaptive cross approximation) [31].

To determine the tensor ml_rank, the MLSVD is performed on all tensors obtained from the first seizure and the seizurefree EEG prior to it for both tensor sets (for more information about the available data see Table II). Then, the obtained singular values (SV) are inspected in order to truncate the MLSVD core where the SV represented more than $95 \%$ of the data variance. The truncated MLSVD core was used to initialize the LMLRA. The LMLRA method is then performed to find the multilinear rank that best approximates the tensor in the least squares sense [31].

As before, this procedure is performed for all tensors obtained from the EEG epochs corresponding to the first seizure and all available seizure-free epochs prior to it. Therefore, for every segment, we obtained a rank value in each of the 3 modes (i.e. frequency, time and space, $\left(R_{f}, R_{t}, R_{c h}\right)$ ). Afterwards, rank histograms are constructed by counting the number of tensors with a certain rank, as shown in Fig.2 Based on these histograms, the most frequently occurring ranks at each mode are selected as upper bound to perform CPD and BTD.

The best tensor model for the classification task is selected by computing the modeling power (MP) and the discrimination power (DP) of the signatures obtained from the CPD and BTD with a certain rank. The MP describes the relevance of the variable for the model and the DP expresses how well a variable discriminates between different classes. The MP and DP were proposed by Wold et. al. in [32] for feature selection in chemical applications. Subsequently, these measures have been used for feature selection in diverse applications as image processing [33], DNA samples selection [34], and biomedical problems modeling [35], [36]. The MP and DP analysis were performed for the signatures obtained from the tensors computed from the EEG epochs corresponding to the first seizure and all available seizure-free epochs prior to it.

Before introducing the DP and MP computation, let us define the feature matrices $H^{A} \in \mathbb{R}^{I_{A} \times F}, H^{B} \in \mathbb{R}^{I_{B} \times T}$ and $H^{C} \in \mathbb{R}^{I_{C} \times C h}$ corresponding to the Frequency, Time and Space modes respectively. $I_{A}=E p \cdot L, I_{B}=E p \cdot M$, 

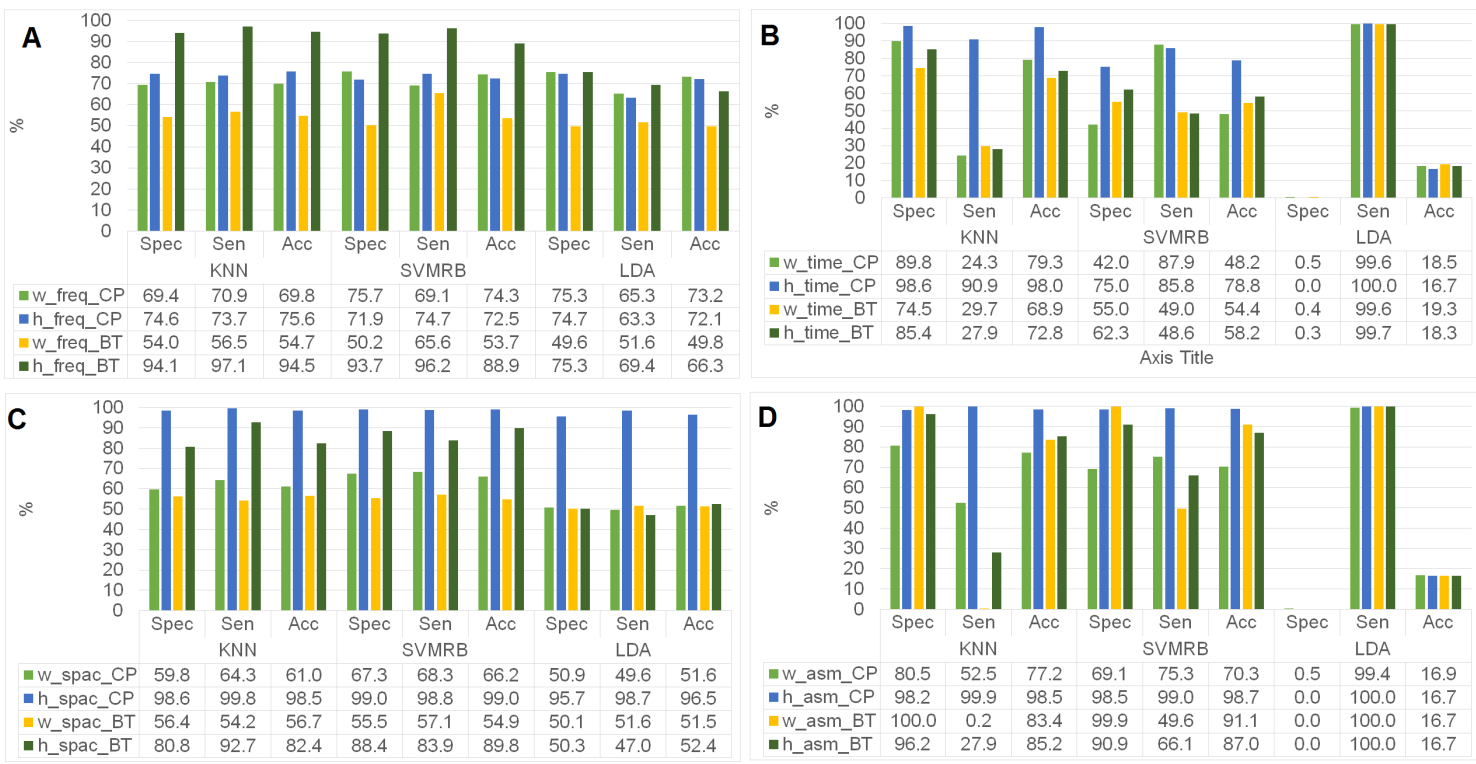

Fig. 4. Sensitivity, Specificity and Accuracy scores obtained in the classification for KNN, SVMRB and LDA.

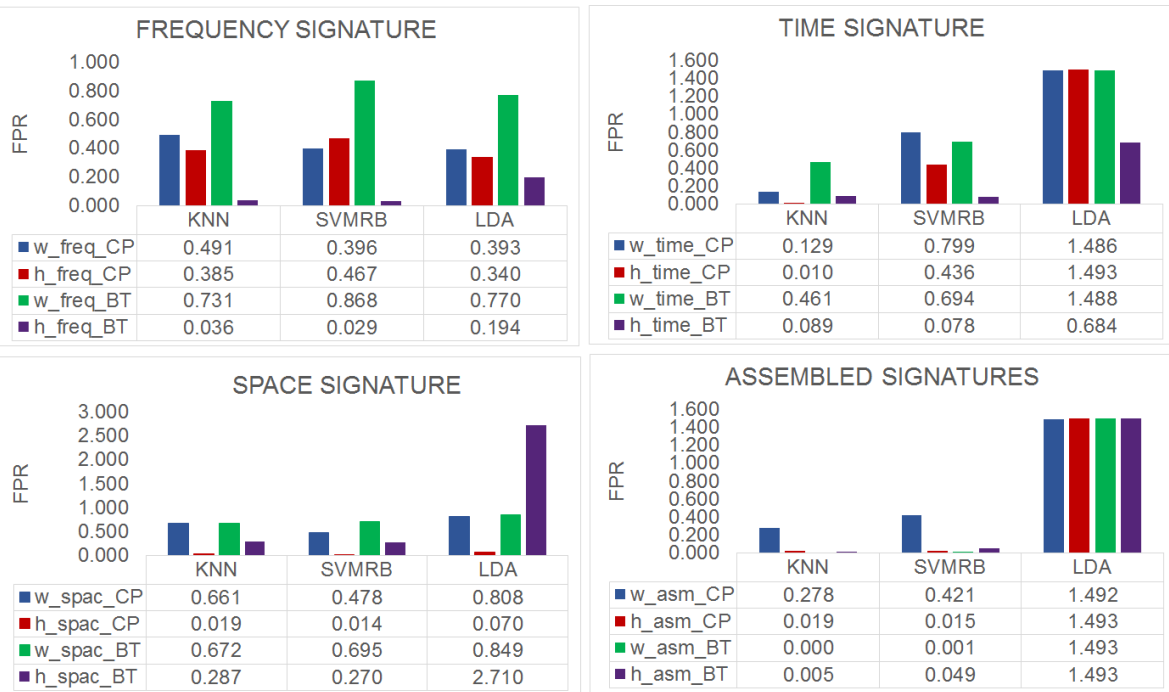

Fig. 5. FPR values displayed by the KNN, SVMRB and LDA classifiers during the different trainings.

and $I_{C}=E p \cdot O$ where, $E p$ is the number of EEG epochs analyzed, $\mathrm{L}, \mathrm{M}$, and $\mathrm{O}$ are the ml-rank and $A_{j} \in \mathbb{R}^{F \times L}$, $B_{j} \in \mathbb{R}^{T \times M}$ and $C_{j} \in \mathbb{R}^{C h \times O}$ are the factor matrices of the Frequency, Time and Space modes respectively, of the $j^{\text {th }}$ epoch, $j=1, \ldots, E p$ with

$$
H^{A}=\left[\begin{array}{c}
A_{1}^{T} \\
A_{2}^{T} \\
\vdots \\
A_{E p}^{T}
\end{array}\right]
$$

and $H^{B}$ and $H^{C}$ similarly defined.

To compute the MP and the DP, at first stage, principal component analysis (PCA) is carried out on the feature matrices $H^{A}, H^{B}$ and $H^{C}$ separately. The number of principal components (PC) $\lambda^{H^{A}}, \lambda^{H^{B}}$ and $\lambda^{H^{C}}$ that represents $95 \%$ of the variance of the feature matrices $H^{A}, H^{B}$ and $H^{C}$ respectively are retained.

Let us take the $H^{A}$ feature matrix to exemplify the MP and DP computation. For more clarity in the notation, let $H^{A}$ be replaced by $H$ and substitute the number of frequency scales $F$ by the index $J$ as the number of columns of $H^{A}$. Then, the MP of the $j^{\text {th }}$ column of $H\left(H_{j}\right)$ is defined as [32] [34]:

$$
M P_{j}^{H}=1-\frac{\sqrt{\sum_{i=1}^{n^{H}} \frac{e_{i j}^{H^{2}}}{\left(n^{H}-\lambda^{H}-1\right)}}}{\sqrt{\sum_{i=1}^{n^{H}} \frac{\left(H_{i j}-\mu_{j}^{H}\right)^{2}}{n^{H}-1}}}
$$

Where $\mu_{j}^{H}$ is the mean of $H_{j}, n^{H}$ is the number of elements in $H_{j}, e_{i j}^{H}$ is the $(i, j)^{t h}$ entry of the residual matrix $E^{H}$ obtained from $\mathrm{H}$ as follows:

$$
E^{H}=H-P_{\lambda^{H}}^{H} Q_{\lambda^{H}}^{H^{T}}
$$




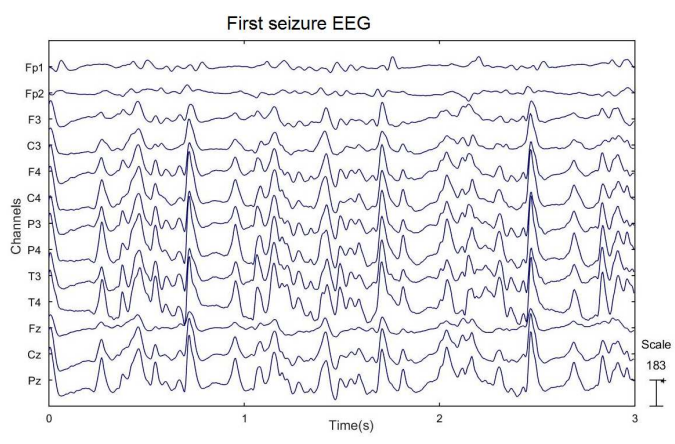

First seizure signatures
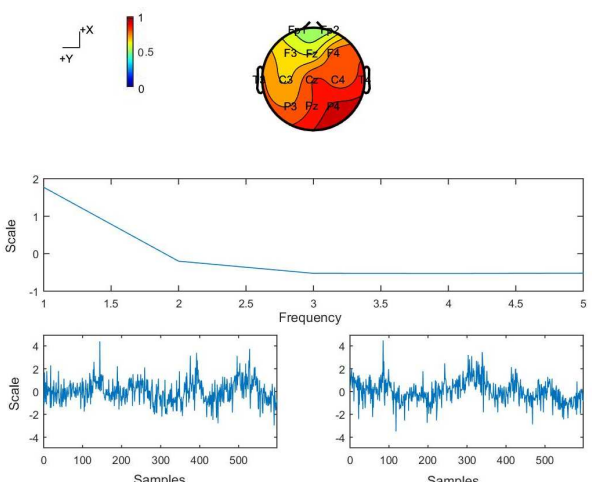

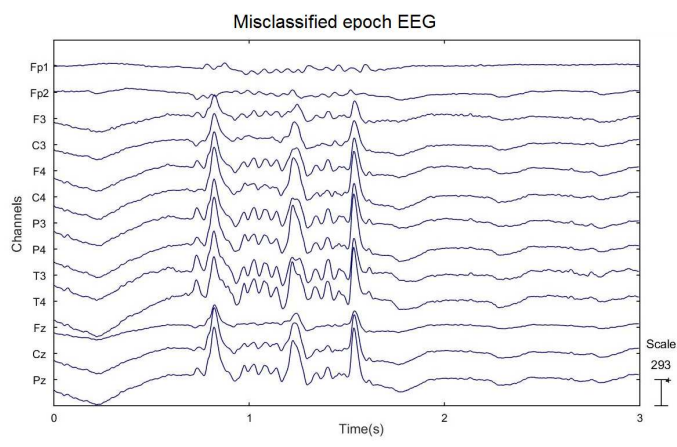

Mistaken epoch signatures
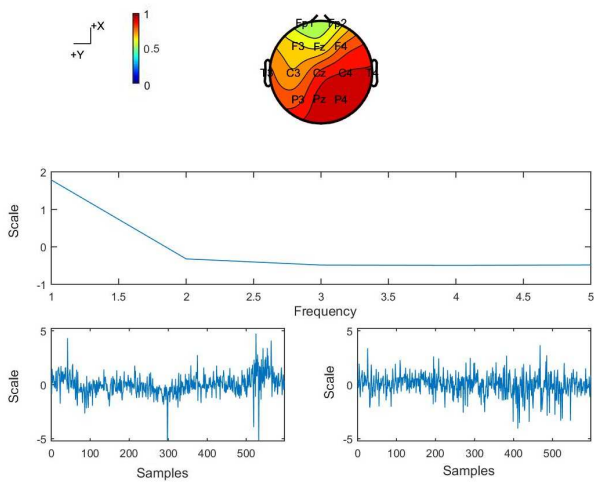

Fig. 6. H_Tensors BTD: false positive classification example. Misclassified seizure-free epoch shows a short paroxysm of approximately 1s of seizure-like activity in the EEG. This activity is captured by highly similar signatures as the first seizures time and frequency signatures. This leads to the detection of the paroxysm as a seizure. Signatures are represented in the following order: Space, Frequency and Time.

Where $P_{\lambda^{H}}^{H}$ are the $\lambda^{H}$ retained PC and $Q_{\lambda^{H}}^{H}$ are the retained PC loadings respectively after truncated PCA.

This measure takes values between 0 and 1 . An MP value close to 1 implies that the variable is relevant for the class description. If this value is close to 0 , then it can be concluded that the variable in terms of modeling is less significant [32].

To compute DP, let us define NCES and non-NCES as two classes $s z=N C E S$ and $n s z=n o n-N C E S$ with feature matrices $H_{s z}$ and $H_{n s z}$ for certain tensor representation. $H_{s z}$ and $H_{n s z}$ are analyzed separately with PCA and the $\lambda^{H_{s z}}$ and $\lambda^{H_{n s z}}$ PCs that represent $95 \%$ of the features variance (for each class) are retained. Then, two PCA models are obtained with $\lambda^{H_{s z}}$ and $\lambda^{H_{n s z}}$ PCs. The DP between the two classes, $s z$ and $n s z$, of the feature $j$ is defined as,

$$
D P_{j}^{H_{s z}, H_{n s z}}=\sqrt{\frac{S_{j}^{H_{n s z}}\left(H_{s z}\right)^{2}+S_{j}^{H_{s z}}\left(H_{n s z}\right)^{2}}{S_{j}^{H_{n s z}}\left(H_{n s z}\right)^{2}+S_{j}^{H_{s z}}\left(H_{s z}\right)^{2}}}-1
$$

Where $S_{j}^{H_{n s z}}\left(H_{n s z}\right)^{2}$ and $S_{j}^{H_{s z}}\left(H_{s z}\right)^{2}$ are the standard deviation computed from the residual matrices $E^{H_{n s z}}$ and $E^{H_{s z}}$ obtained for $H_{n s z}$ and $H_{s z}$ PCA models with (15), and are defined as,

$$
S_{j}^{H_{n s z}}\left(H_{n s z}\right)^{2}=\sum_{i=1}^{n^{H_{n s z}}} \frac{e_{i j}^{H_{n s z}{ }^{2}}}{\left(n^{H_{n s z}}-\lambda^{H_{n s z}}-1\right)}
$$

and

$$
S_{j}^{H_{s z}}\left(H_{s z}\right)^{2}=\sum_{i=1}^{n^{H_{s z}}} \frac{e_{i j}^{H_{s z}{ }^{2}}}{\left(n^{H_{s z}}-\lambda^{H_{s z}}-1\right)}
$$

where $\lambda^{H_{n s z}}$ and $\lambda^{H_{s z}}$ are the number of retained PCs for $H_{n s z}$ and $H_{s z}$ PCA models respectively. The $S_{j}^{H_{n s z}}\left(H_{s z}\right)^{2}$ and $S_{j}^{H_{s z}}\left(H_{n s z}\right)^{2}$ terms are the residual standard deviation of $H_{n s z}$ and $H_{s z}$ when fitting samples from the class $H_{s z}$ onto the PCA model obtained for class $H_{n s z}$, and when fitting samples from the class $H_{n s z}$ onto the PCA model obtained for class $H_{s z}$ respectively [32], these terms are defined as,

$$
S_{j}^{H_{s z}}\left(H_{n s z}\right)^{2}=\frac{J}{n^{H_{n s z}}\left(J-\lambda^{H_{s z}}\right)} \sum_{i=1}^{n^{H_{n s z}}}\left(e_{i j}^{H_{n s z}}\right)^{2}
$$

and

$$
S_{j}^{H_{n s z}}\left(H_{s z}\right)^{2}=\frac{J}{n^{H_{s z}}\left(J-\lambda^{H_{n s z}}\right)} \sum_{i=1}^{n^{H_{s z}}}\left(e_{i j}^{H_{s z}}\right)^{2}
$$

Where $J$ is the number of columns of $H_{n s z}$ and $H_{s z}, n^{H_{s z}}$ and $n^{H_{n s z}}$ are the number of rows of $H_{n s z}$ and $H_{s z}$ respectively.

If the DP value is close to 0, low discriminatory power is observed. If it is much higher than 1 , a good discriminatory power is observed [32].

Fig.3 shows the computed MP and DP values for the signatures obtained from HH_Tensor and W_Tensor after 

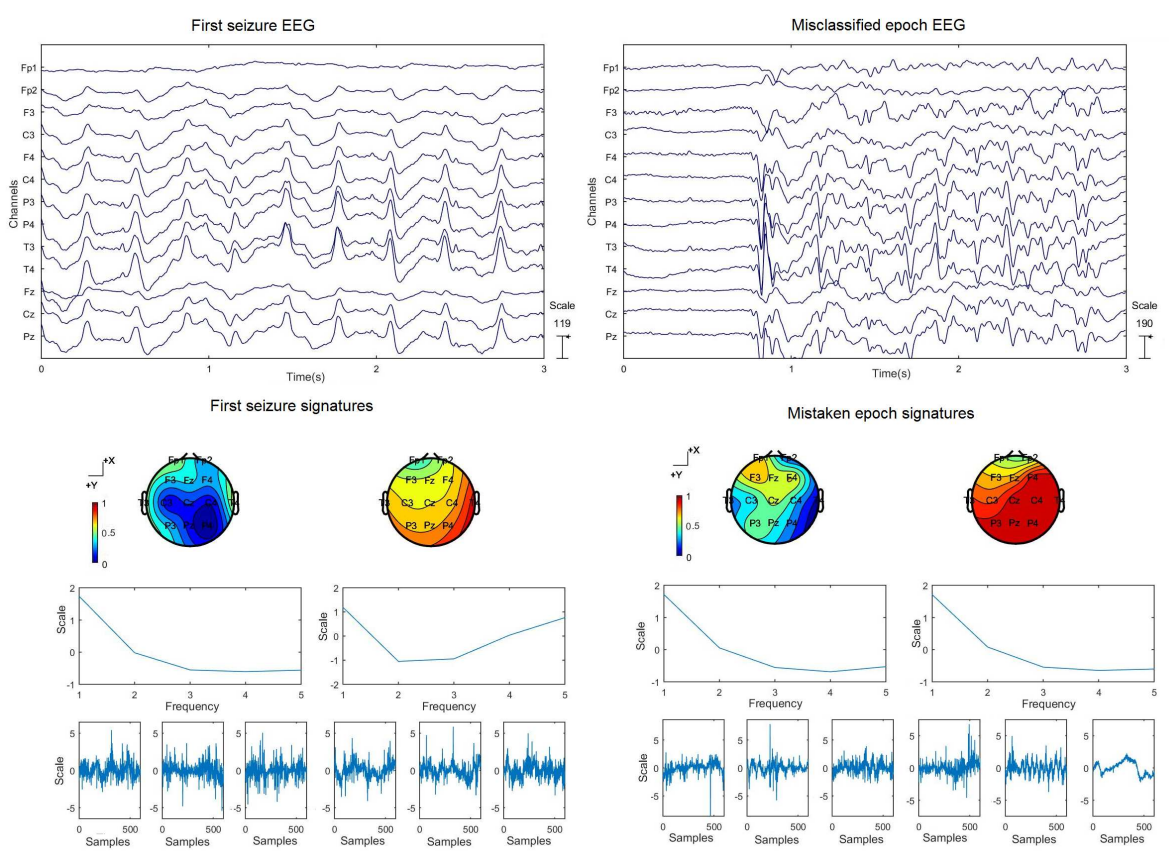

Fig. 7. W Tensors BTD: false negative classification example. Seizure epoch classed as a non-seizure. The EEG seizure patterns from the misclassified epoch are dissimilar to the patterns from the first seizure. The space signature from the misclassified epoch shows a maximum activation pattern which is more spread than the showed by first seizure, giving an idea of a more violent crisis. The second frequency component from the first seizure shows a rise in the frequency values at $5 \mathrm{~Hz}$ while the second component from the misclassified epoch maximum is around $1 \mathrm{~Hz}$. The time signatures are more difficult to interpret by visual inspection. However, it is possible to note that there are some differences between the ones from the first seizure and the ones from the misclassified epoch. These dissimilarities explain the classifier failure. Signatures are represented in the following order: Space, Frequency and Time.

applying CPD. Based on the LMLRA (see Fig.2) the element of the ml-rank triplet $\left(R_{f}, R_{t}, R_{c h}\right)$ most commonly occurring at each mode is inspected. Then, the largest rank among the three modes, denoted as $R$, is selected as an upper bound to perform CPD. This led to performing CPD with $R=1,2,3$ for HH_Tensor and $R=2, \ldots, 6$ for W_Tensor. BTD is applied to all rank combinations of $R_{f}=1,2, R_{t}=1,2,3$ and $R_{c h}=1,2,3$ for HH_Tensor and $R_{f}=2, R_{t}=4,6$ and $R_{c h}=2,3$ for W_Tensor (where $R_{f}, R_{t}$, and $R_{c h}$ are the possible rank values of the Frequency, Time and Space modes respectively). This results in a total of 17 different tensor representations for HH_Tensor (excluding the ml_rank$(1,1,1)$ since it is equivalent to an $R=1 \mathrm{CPD})$ and 4 tensor representations for $\mathrm{W} \_$Tensor.

The average MP value is computed by averaging the values obtained by the three signatures for each tensor representation. The same procedure is performed for the DP. Then, the tensor representation with the average MP closest to 1 and the largest DP (over 1) is chosen. From this analysis, it is decided to decompose the W_Tensor set with $m l$-rank- $(2,6,2)$ and $R=1$ for BTD and $R=2$ for CPD. For HH_Tensor BTD analysis is performed with ml-rank- $(1,2,1)$ and $R=1$ for both BTD and CPD.

Note that in the case of HH_Tensor, the maximum average MP and DP values of the signatures for CPD are achieved for different tensor representations. The maximum average MP is obtained for $R=2$ with $M P=0.68$ and $D P=3.82$ and the maximum average DP is obtained for $R=1$ with $M P=0.66$ and $D P=5.58$. As can be appreciated the difference in MP values for these tensor representations is small compared to the difference in DP values. Since the task to be solved is a classification task, we do not look for an exact approximation of the data but a sufficiently good approximation that allows separating between seizure and non-seizure data. Therefore, the $\mathrm{R}$ with higher average DP value is selected.

\section{E. Classification}

Three classifiers are used to perform the NCES detection: A K-Nearest Neighbor classifier (KNN) with $K=3$, a Radial Basis Support Vector Machine(SVMRB), and a Linear Discriminant Analysis (LDA). The implementation used for the SVMRB estimates automatically the optimal radial basis kernel and the regularization parameter. The kernel optimization is performed by quadratic programming using the QP solver provided by MatLab. The regularization parameter is estimated by the leave-one-out cross-validation. The classifier selection is based on a previous study [37] where it was concluded that these are the best classifiers when using tensor-based signatures as features. The KNN and SVMRB implementation are available at the MATLAB toolbox PRTools [38].

The classification is performed for each patient individually. The training set for each case is assembled with the epochs from the first seizure (= positive class) and the same number of non-seizure epochs from the EEG prior to the first seizure (=negative class). Details about the training sets are given in Table II. It was impossible to balance the training sets for the cases 2 and 3 since the duration of the EEG prior to the first seizure was too short to meet the number of epochs from the seizure (the first seizure started after a few seconds of 
recording). Yet, this does not seem to affect the classifier performance. The classification was performed in two ways: (1) with the signatures from the different modes (Frequency, Time, Space) separately, (2) with all mode signatures assembled.

The classifiers' performance is evaluated in terms of sensitivity, specificity, accuracy and false negative rate. Where the sensitivity is calculated as,

$$
\text { Sen }=\frac{T P}{T P+F N}
$$

The specificity is defined as,

$$
\text { Spec }=\frac{T N}{T N+F P}
$$

The accuracy is termed as,

$$
A c c=\frac{T P+T N}{T P+F P+T N+F N}
$$

The false positive rate denotes the Type I error ratio (false epochs classified as positives) and is defined as

$$
F P R=\frac{F P}{F P+T N}
$$

Where TP is the number of seizure epochs correctly detected, FP is the number of seizure-free epochs classified as seizure, $\mathrm{TN}$ is the number of seizure-free epochs correctly detected and $\mathrm{FN}$ is the number of missed seizure epochs.

\section{RESUlTS AND Discussion}

The classification results are presented for the epoch based analysis since the duration of the epochs is the minimum duration of an epileptic event in the EEG. This means that an alarm should be displayed after each positive detection. This is not a practical implementation in real life dynamical setting. For the real-life setting, an alarm will be set at the seizure activity onset. Then, subsequent alarms are suppressed and a new alarm is allowed after 10 minutes (lower bound established for NCSE diagnosis [1]) of continuous seizure activity.

The classifiers' performance for the Frequency signatures is shown in Fig.4 A. The best classification results are obtained by a KNN classifier for h_freq_BT signatures with a 94.1, 97.1 , and $94.15 \%$ of specificity, sensitivity and accuracy values respectively, and with a false detection rate per hour of 0.036 . According to the analysis of MP values, Frequency signatures offer the best model of the data when they are obtained from the decomposition of the $\mathrm{W}_{-}$Tensor set either with CPD or BTD. On the other hand, the highest DP is obtained for $\mathrm{h}$ ffreq_ BT signatures followed by the $\mathrm{h}$ _freq_CP signatures. This analysis revealed that even if the signatures allow an accurate prediction model of the original data, as is the case for W_Tensors signatures, discrimination between classes is not guaranteed. The rest of the Frequency signatures achieved sensitivity and accuracy values under $80 \%$.

Regarding the Time feature, the highest DP value is obtained with h_time_CP signatures. Consequently, the best classification results for this feature are achieved by h_time_CP signatures with a KNN classifier (see Fig.4 B). The classifier reached $98.6,90.9$ and $98.0 \%$ of specificity, sensitivity and accuracy values respectively. This feature achieved a false positive detection rate of 0.010 .

The false positive detection number for the Time feature increases for the h_time_BT, w_time_CP, and w_time_BT signatures. This could be explained by the increase in the number of factors used for these signatures. The greater the number of factors, the more uninteresting patterns are modeled by the signatures, which increases the uncertainty in the classification task.

The combination of the signatures obtained with CPD and BTD is unique. Yet, the components computed (signatures) on their own are not. When more than one component is computed, it is expected that some of them fit the pattern of interest (hopefully most of them), and others model the noise in the data or other underlying patterns that may appear. The order in which these components are obtained is not unique and may vary from epoch to epoch. It is not possible to determine which component contains NCES information based on it. Hence, the features computed from different epochs may not correspond to each other, thereby hampering the classification based only on the signatures.

We further discuss the Space signature performance. As low DP values were obtained for W_Tensors CPD and BTD space signatures, the classifier performance for these signatures is lower than that obtained for the HH_Tensors signatures, Fig.4 C. The highest specificity, sensitivity and accuracy values for the Space feature are achieved by SVMRB with $99.0 \%, 98.8 \%$ and $99.0 \%$ respectively for the h_spac_CP signatures.The KNN classifier obtained similar results for these signatures with a specificity of $98.6 \%$, a sensitivity of $99.8 \%$, and accuracy of $98.5 \%$. The h_spac_CP signatures combined with SVMRB achieved a 0.014 as false positive detection rate value per hour.

The KNN classifier achieved sensitivity and accuracy values below $85 \%$ for h_spac_BT. It is expected to obtain lower sensitivity and accuracy values for $\mathrm{h} \_$spac_BT signatures given that the DP for these is 3.9 compared to 6.4 computed for h_spac_CP. Yet, the SVMRB classifier obtained accuracy and sensitivity values over $85 \%$ outperforming KNN. Even when lower performance scores are expected for h_spac_BT based on the DP values, these should not be as low as those obtained for $\mathrm{KNN}$, since 3.9 is not a negligible DP value. Hence, a higher performance could be expected from KNN. The inspection of the h_spac_BT signatures showed that the scatter distribution of these resulted in more overlap between the classes, explaining KNN's poor performance.

The training set using the assembled signatures achieved similar results to those achieved by the space signature. The best classifier for the assembled signatures training is SVMRB for HH_Tensor CPD signatures with a specificity value of $98.5 \%$, a sensitivity value of $99.0 \%$ and accuracy of $98.7 \%$. The average DP computed for these signatures is 5.5 , it is lower than the 7.5 obtained for h_ass_BT signatures. Yet, if the DP values are analyzed individually for each signature, it can be noticed that $h$ _freq_BT displays a large DP of 21.9 while the DP values of the remaining $h$ _time_BT and h_spac_BT are low, 2.2 and 2.6 respectively. The DP values for HH_Tensor_CPD are 5.9, 4.4 and 6.4 for h_freq_CP, 
TABLE II

AVAILABLE DATA FOR NCES AND SEIZURE FREE (N-NCES) ClASS AND THE RESULTING TRAINING SETS FOR EACH EEG RECORD GIVEN IN EPOCHS OF 3S DURATION

\begin{tabular}{|c|c|c|c|c|c|c|c|c|}
\hline \multirow[b]{4}{*}{ Record } & \multicolumn{8}{|c|}{ Epochs } \\
\hline & \multirow{2}{*}{\multicolumn{2}{|c|}{ Available Data }} & \multicolumn{4}{|c|}{ Fist Seizure and prior EEG } & \multirow{2}{*}{\multicolumn{2}{|c|}{ Test Set }} \\
\hline & & & \multicolumn{2}{|c|}{ Modeling Data } & \multicolumn{2}{|c|}{ Training Set } & & \\
\hline & NCES & n-NCES & NCES & n-NCES & NCES & n-NCES & NCES & $\mathrm{n}$-NCES \\
\hline 1 & 58 & 345 & 4 & 177 & 4 & 4 & 54 & 168 \\
\hline 2 & 39 & 127 & 26 & 5 & 26 & 5 & 13 & 122 \\
\hline 3 & 140 & 424 & 12 & 1 & 12 & 1 & 128 & 423 \\
\hline 4 & 73 & 112 & 8 & 30 & 8 & 8 & 65 & 82 \\
\hline 5 & 10 & 18 & 3 & 7 & 3 & 3 & 7 & 11 \\
\hline 6 & 84 & 269 & 13 & 18 & 13 & 13 & 77 & 251 \\
\hline 7 & 6 & 237 & 3 & 143 & 3 & 3 & 3 & 94 \\
\hline 8 & 13 & 4827 & 3 & 18 & 3 & 3 & 7 & 4809 \\
\hline 9 & 163 & 4558 & 3 & 24 & 3 & 3 & 160 & 4534 \\
\hline 10 & 1074 & 3346 & 19 & 125 & 19 & 19 & 1055 & 3221 \\
\hline 11 & 16 & 1841 & 12 & 1381 & 12 & 12 & 4 & 460 \\
\hline 12 & 875 & 1036 & 87 & 152 & 87 & 87 & 788 & 884 \\
\hline 13 & 90 & 860 & 16 & 33 & 16 & 16 & 74 & 827 \\
\hline 14 & 44 & 472 & 13 & 59 & 13 & 13 & 31 & 413 \\
\hline Total & 2685 & 18472 & 222 & 2173 & 222 & 190 & 2466 & 16299 \\
\hline
\end{tabular}

h_time_CP and h_spac_CP respectively. Thus, this implies that the assembled training results will depend directly on the individual signatures quality. The false detection rate for the assembled HH_Tensor CPD training is similar to the one obtained by the h_spac_CP signatures training.

The performed experiments showed that the tensor built with HHT separates better the NCES data, thereby outperforming the tensor obtained with the WT. Note that we also tested datasets created using the energy of the wavelets coefficients and other mother wavelets. None of these data sets obtained better results in the training than those obtained using the wavelet coefficients computed with the Mexican Hat mother wavelet or HHT.

The HH_Tensor signatures obtained with CPD outperform those computed with BTD in most of the training sets. This is somewhat unexpected, as NCES is known to evolve along the EEG. A low-rank BTD is a suitable model for analyzing such evolving patterns [19]. The NCES can evolve along the EEG record in frequency, morphology (time), or location (space). The Salzburg Consensus Criteria for NCSE proposed in [22] by adopting the American Clinical Neurophysiology Society (ACNS) criterion provided more detailed, unambiguous guidelines about the characteristics of this "evolution" in each of these dimensions which could clarify this unexpected result. According to the ACNS, evolution in frequency is defined as at least 2 consecutive changes in the same direction by at least $0.5 \mathrm{~Hz}$, e.g. from 2 to 2.5 to $3 \mathrm{~Hz}$, or from 3 to 2 to $1.5 \mathrm{~Hz}$; Evolution in morphology implies at least 2 consecutive changes to a novel morphology; Evolution in location is defined as sequentially spreading into or sequentially out of at least two different standard 10-20 electrode locations. In order to qualify as "present", a single frequency or location must persist at least $3 \mathrm{~s}$ [22]. This implies that the changes usually occur slowly, and a certain pattern tends to persist $3 \mathrm{~s}$ or longer. The CPD model assumes that the source data preserve the same frequency and location within the observed epoch [19]. Due to the analyzed epochs duration (3s), it is therefore unlikely to observe a lot of evolution in frequency, time or space occurring within the same epoch. Hence, the NCES data analyzed in these small epochs fulfill the trilinear criteria justifying CPD and explaining why BTD overfits the model.

Although HHT and WT are used for this proposal, different tensorizations would also be possible, e.g. empirical wavelet transform and tunable-Q wavelet transform [39], [40], which were successfully used to detect epileptic seizures. Such methods could be tried on NCES data in order demonstrate if these algorithms are capable of capturing this seizure activity as well.

Both classifiers, KNN and SVMRB, showed similar performance for classification for different training sets. However, different combinations of signatures and classifiers performed better for the different scenarios (e.g. h_spac_BT combined with SVMRB outperform the combination of $h \_s p a c \_B T$ and KNN, h_freq_BT combined with KNN outperform h_freq_BT combined with SVMRB). Therefore, is not possible to establish a clear superiority of one over the other given the obtained results.

The LDA classifier showed to be able to classify NCES data with the Frequency and Space signatures. This classifier obtained values of specificity, sensitivity, and accuracy over 95\% for the h_spac_CP. However, for high dimensional features such as Time and Assembled signatures LDA showed to be unable to identify the seizure from seizure-free data. For the Time and Assembled signatures, LDA displayed high sensitivity but low values of specificity and accuracy.

As the achieved false positive rates values suggest, Fig.5, the number of non-seizure events classified as seizures is low. The majority of these misclassifications are due to the appearance of long duration paroxysms (Fig.6) or a large number of successive spikes in the EEG. These events, are long enough to resemble a seizure but are too brief to be classified as a seizure 
according to the physician's criteria. False positives detections also occur at epochs with pre-ictal or post-ictal EEG activity.

False negative detections are found in records longer than 10 hours. These are related to changes in the EEG patterns of the most distant seizures in time compared to the first detected seizure (Fig.7). These changes may be associated with the patient's disease evolution. Including new EEG patterns from subsequent seizures to the initial training set could help to solve this. This will make the method proposed here robust to the changes in the seizure pattern. There is no need to stop the classification to retrain the classifier, this can be done in parallel. This is a strength comparing our proposal to threshold-based methods. The threshold based methods are unable to respond to seizure changes and background EEG pattern changes. This lack of adaptability makes the threshold based methods less accurate for long-term EEG recordings.

Some misclassifications are caused by high-frequency artifacts probably related to poor electrode contact. These are only found at the epochs where the artifact affected more than one electrode at the same time instant. All signatures proved to be robust to artifacts affecting isolated electrodes.

Based on the performed analysis, the best combination for NCES detection is provided by the Space signatures as features and the SVMRB as classifier. The Space signature must be obtained with HHT and CPD as tensorization and tensor decomposition method respectively.

TABLE III

ROUGH COMPARISON OF THE PROPOSED METHOD PERFORMANCE WITH OTHER EXISTING METHODS IN TERMS OF ACCURACY, SENSITIVITY AND SPECIFICITY

\begin{tabular}{|c|c|c|c|}
\hline Method & Accuracy & Sensitivity & Specificity \\
\hline Proposed Method & $99.0 \%$ & $98.8 \%$ & $99.0 \%$ \\
Liang et. al. [14] & $97.5 \%$ & $\mathrm{~N} / \mathrm{R}$ & $\mathrm{N} / \mathrm{R}$ \\
Xanthopoulos et. al. [12] & $95.0 \%$ & $\mathrm{~N} / \mathrm{R}$ & $\mathrm{N} / \mathrm{R}$ \\
Kollialil et. al. [13] & $99.6 \%$ & $\mathrm{~N} / \mathrm{R}$ & $\mathrm{N} / \mathrm{R}$ \\
Fatma et. al. [41] & $88.2 \%$ & $\mathrm{~N} / \mathrm{R}$ & $\mathrm{N} / \mathrm{R}$ \\
Jacquin et. al. [11] & $\mathrm{N} / \mathrm{R}$ & $83.0 \%$ & $96.0 \%$ \\
Minasyan et. al. [15] & $\mathrm{N} / \mathrm{R}$ & $71.0 \%$ & $99.0 \%$ \\
Khan et. al. [16] & $\mathrm{N} / \mathrm{R}$ & $86.8 \%$ & $96.9 \%$ \\
Sharma et. al. [18] & $\mathrm{N} / \mathrm{R}$ & $100.0 \%$ & $93.3 \%$ \\
Fatma et. al. [41] & $\mathrm{N} / \mathrm{R}$ & $100.0 \%$ & $88.0 \%$ \\
\hline
\end{tabular}

Table III presents a rough comparison of our results with the results achieved by other methods for NCES detection, in terms of sensitivity and specificity. As can be noticed, the methods from Sharma et. al. [16] and Fatma et. al. [41] obtained superior results in terms of sensitivity than those achieved by the method proposed here. The database used in these publications consists of 13 EEG records, 6 of which have only one seizure. The parameters extracted from epochs of the same seizure tend to display a high similarity. Hence, any classification task made with parameters from epochs of the same seizure will reach high sensitivity values.

In terms of accuracy, Table III shows that our method is only outperformed by Kollialil et. al. [11]. The method proposed by Kollialil uses an SVM to classify between normal, interictal and seizure EEG. In this publication, three different labeled datasets are used. The classification was not performed in a continuous recording. It should be noted that with such test data, the classifier does not face issues as artifacts, EEG pattern changes or paroxysms as done in our study.

Regarding the execution time, which is a major concern for detection algorithms, our method displays a runtime from $6.04 \mathrm{~s}$ to $3.3 \mathrm{~min}$ for the training step. All tests are performed on a computer with an Intel Core-i3 processor at $1.70 \mathrm{GHz}$ with $8 G B$ of RAM. The training results are obtained for the EEG records with the shortest $(9 \mathrm{~s})$ and the largest $(4.35 \mathrm{~min})$ first seizure respectively. It should be noted, that the training will only be performed once at the beginning of the monitoring. The training step includes the tensorization and analysis of all first seizure epochs and the classifier training. Hence, the execution time will depend on the first seizure duration. It could be decided to use only part of the seizure for training if this is very long. Yet, it should be kept in mind that doing so fewer training patterns will be included. In this sense, there is a compromise between the number of positive epochs to include in the training set and the quality of the classifier.

The classification runtime ranged from 0.37 to $3 s$ which means that our method can run in real-time. These results are obtained by classifying all epochs from the test set. The duration of the classification step will depend on the pattern complexity. As the pattern becomes more complex, more time is required to perform the EMD. The average detection delay of the algorihtm is $5.4 \mathrm{~s}$.

\section{CONCLUSION}

The presented paper introduced a NCES detection method that exploits techniques such as multiway data analysis and HHT. It is the first algorithm to apply HHT to model seizure data with tensors. This is the first algorithm that uses this combination for NCES detection. The HHT provides an accurate definition of particular events in the time $\times$ frequency space capturing the NCES data in a tensor close to $R=1$. This turns all signatures obtained from $\mathrm{HH}$ _Tensor decomposition in relevant features to perform the NCES detection.

The best results at the classification were achieved for HH_Tensor Space and Assembled features computed with CPD combined with the SVMRB classifier, closely followed by HH_Tensor Frequency features computed with BTD combined with KNN.

According to the obtained results, the proposed method proved to be one of the best NCES methods so far. The method was validated on continuous clinical data in a realistic training setting proving that the method can run in real-time.

The number of false positives for the training with HH_Tensor Space and Assembled signatures obtained with CPD was low. Yet, the appearance of EEG activity resembling the seizure affected the performance of these signatures. The false negatives found were related to EEG pattern changes at the seizures distant in time from the first recorded seizure. Due to the seizure EEG pattern evolution caused by the underlying disease progression, adaptive training strategies must be implemented to improve this.

\section{REFERENCES}

[1] E. Trinka, H. Cock, D. Hesdorffer, A. O. Rossetti, I. E. Scheffer, S. Shinnar, S. Shorvon, and D. H. Lowenstein, "A definition and classification 
of status epilepticus-report of the ilae task force on classification of status epilepticus," Epilepsia, vol. 56, no. 10, pp. 1515-1523, 2015.

[2] P. M. Vespa, V. Nenov, and M. R. Nuwer, "Continuous eeg monitoring in the intensive care unit: early findings and clinical efficacy," Journal of Clinical Neurophysiology, vol. 16, no. 1, pp. 1-13, 1999.

[3] D. L. Schomer and F. L. Da Silva, Niedermeyer's electroencephalography: basic principles, clinical applications, and related fields. Lippincott Williams \& Wilkins, 2012.

[4] A. T. Tzallas, D. G. Tsalikakis, E. C. Karvounis, L. Astrakas, M. Tzaphlidou, M. G. Tsipouras, and S. Konitsiotis, Automated epileptic seizure detection methods: a review study. Citeseer, 2012.

[5] L. Orosco, A. G. Correa, and E. Laciar, "Review: a survey of performance and techniques for automatic epilepsy detection," Journal of Medical and Biological Engineering, vol. 33, no. 6, pp. 526-537, 2013.

[6] A. Q. Ansari and P. Sharma, "A review on automated detection of nonconvulsive seizures using eeg," in Computational Intelligence \& Communication Technology (CICT), 2016 Second International Conference on. IEEE, 2016, pp. 283-286.

[7] H. Adeli, Z. Zhou, and N. Dadmehr, "Analysis of eeg records in an epileptic patient using wavelet transform," Journal of neuroscience methods, vol. 123, no. 1, pp. 69-87, 2003.

[8] A. Jacquin, E. Causevic, and E. R. John, "Automatic identification of spike-wave events and non-convulsive seizures with a reduced set of electrodes," in Engineering in Medicine and Biology Society, 2007. EMBS 2007. 29th Annual International Conference of the IEEE. IEEE, 2007, pp. 1928-1931.

[9] P. Xanthopoulos, S. Rebennack, C.-C. Liu, J. Zhang, G. L. Holmes, B. M. Uthman, and P. M. Pardalos, "A novel wavelet based algorithm for spike and wave detection in absence epilepsy," in BioInformatics and BioEngineering (BIBE), 2010 IEEE International Conference on. IEEE, 2010, pp. 14-19.

[10] S.-F. Liang, W.-L. Chang, and H. Chiueh, "Eeg-based absence seizure detection methods," in Neural Networks (IJCNN), The 2010 International Joint Conference on. IEEE, 2010, pp. 1-4.

[11] E. S. Kollialil, G. K. Gopan, A. Harsha, and L. A. Joseph, "Single feature-based non-convulsive epileptic seizure detection using multiclass svm," in Emerging Trends in Communication, Control, Signal Processing \& Computing Applications (C2SPCA), 2013 International Conference on. IEEE, 2013, pp. 1-6.

[12] G. R. Minasyan, J. B. Chatten, and R. N. Harner, "Detection of epileptiform activity in unresponsive patients using ann," in Neural Networks, 2009. IJCNN 2009. International Joint Conference on. IEEE, 2009 , pp. $2117-2124$

[13] J. C. Sackellares, D.-S. Shiau, J. J. Halford, S. M. LaRoche, and K. M. Kelly, "Quantitative eeg analysis for automated detection of nonconvulsive seizures in intensive care units," Epilepsy \& Behavior, vol. 22, pp. S69-S73, 2011

[14] Y. U. Khan, O. Farooq, M. Tripathi, P. Sharma, and P. Alam, "Automatic detection of non-convulsive seizures using ar modeling," in Power, Control and Embedded Systems (ICPCES), 2012 2nd International Conference on. IEEE, 2012, pp. 1-4.

[15] S. Varshney, Y. U. Khan, O. Farooq, P. Sharma, and M. Tripathi, "Latency study of non-convulsive seizures," in Multimedia, Signal Processing and Communication Technologies (IMPACT), 2013 International Conference on. IEEE, 2013, pp. 108-111.

[16] P. Sharma, Y. U. Khan, O. Farooq, M. Tripathi, and H. Adeli, "A waveletstatistical features approach for nonconvulsive seizure detection," Clinical EEG and neuroscience, vol. 45, no. 4, pp. 274-284, 2014.

[17] H. Qu and J. Gotman, "A seizure warning system for long-term epilepsy monitoring," Neurology, vol. 45, no. 12, pp. 2250-2254, 1995.

[18] E. Acar, C. Aykut-Bingol, H. Bingol, R. Bro, and B. Yener, "Multiway analysis of epilepsy tensors," Bioinformatics, vol. 23, no. 13, pp. 10-18, 2007.

[19] B. Hunyadi, D. Camps, L. Sorber, W. Van Paesschen, M. De Vos, S. Van Huffel, and L. De Lathauwer, "Block term decomposition for modelling epileptic seizures," EURASIP Journal on Advances in Signal Processing, vol. 2014, no. 1, p. 139, 2014.

[20] W. Deburchgraeve, P. J. Cherian, M. De Vos, R. M. Swarte, J. H Blok, G. H. Visser, P. Govaert, and S. Van Huffel, "Neonatal seizure localization using parafac decomposition," Clinical Neurophysiology, vol. 120, no. 10, pp. 1787-1796, 2009.

[21] C. Guerrero-Mosquera, M. Verleysen, and A. N. Vazquez, "Eeg feature selection using mutual information and support vector machine: A comparative analysis," in Engineering in Medicine and Biology Society (EMBC), 2010 Annual International Conference of the IEEE. IEEE, 2010, pp. 4946-4949.
[22] M. Leitinger, S. Beniczky, A. Rohracher, E. Gardella, G. Kalss, E. Qerama, J. Höfler, A. H. Lindberg-Larsen, G. Kuchukhidze, J. Dobesberger et al., "Salzburg consensus criteria for non-convulsive status epilepticus-approach to clinical application," Epilepsy \& Behavior, vol. 49, pp. 158-163, 2015.

[23] N. E. Huang, Z. Shen, S. R. Long, M. C. Wu, H. H. Shih, Q. Zheng, N.C. Yen, C. C. Tung, and H. H. Liu, "The empirical mode decomposition and the hilbert spectrum for nonlinear and non-stationary time series analysis," in Proceedings of the Royal Society of London A: Mathematical, Physical and Engineering Sciences, vol. 454, no. 1971. The Royal Society, 1998, pp. 903-995.

[24] N.-F. Chang, T.-C. Chen, C.-Y. Chiang, and L.-G. Chen, "On-line empirical mode decomposition biomedical microprocessor for hilbert huang transform," in Biomedical Circuits and Systems Conference (BioCAS), 2011 IEEE. IEEE, 2011, pp. 420-423.

[25] E. A. Atamon, Understanding epilepsy seizure structure using tensor analysis. Rensselaer Polytechnic Institute, 2008.

[26] R. Zink, B. Hunyadi, S. Van Huffel, and M. De Vos, "Tensor-based classification of an auditory mobile bci without a subject-specific calibration phase," Journal of neural engineering, vol. 13, no. 2, p. 026005, 2016.

[27] M. Sørensen and L. De Lathauwer, "Tensor decompositions with blocktoeplitz structure and applications in signal processing," in Signals, Systems and Computers (ASILOMAR), 2011 Conference Record of the Forty Fifth Asilomar Conference on. IEEE, 2011, pp. 454-458.

[28] N. D. Sidiropoulos and R. Bro, "On the uniqueness of multilinear decomposition of n-way arrays," Journal of chemometrics, vol. 14, no. 3, pp. 229-239, 2000.

[29] L. De Lathauwer and D. Nion, "Decompositions of a higher-order tensor in block termspart iii: Alternating least squares algorithms," SIAM journal on Matrix Analysis and Applications, vol. 30, no. 3, pp. 10671083, 2008.

[30] L. De Lathauwer, B. De Moor, and J. Vandewalle, "On the best rank-1 and rank-(r 1, r 2,..., rn) approximation of higher-order tensors," SIAM journal on Matrix Analysis and Applications, vol. 21, no. 4, pp. 1324$1342,2000$.

[31] A. Cichocki, D. Mandic, L. De Lathauwer, G. Zhou, Q. Zhao, C. Caiafa, and H. A. Phan, "Tensor decompositions for signal processing applications: From two-way to multiway component analysis," IEEE Signal Processing Magazine, vol. 32, no. 2, pp. 145-163, 2015.

[32] S. Wold and M. SJÖSTRÖM, "Simca: a method for analyzing chemical data in terms of similarity and analogy." ACS Publications, 1977.

[33] S. Dabbaghchian, M. P. Ghaemmaghami, and A. Aghagolzadeh, "Feature extraction using discrete cosine transform and discrimination power analysis with a face recognition technology," Pattern Recognition, vol. 43 , no. 4 , pp. 1431-1440, 2010.

[34] I. T. Bustamante, F. S. Mata, N. H. González, R. G. Gazapo, J. Palau, and M. M. C. Ferreira, "Application of chemometric tools for automatic classification and profile extraction of dna samples in forensic tasks," Analytica chimica acta, vol. 595, no. 1, pp. 43-50, 2007.

[35] S. Bicciato, A. Luchini, and C. Di Bello, "Pca disjoint models for multiclass cancer analysis using gene expression data," Bioinformatics, vol. 19 , no. 5 , pp. $571-578,2003$.

[36] E. A. Kanık, G. O. Temel, S. Erdoğan, and İ. E. Kaya, "Affected states soft independent modeling by class analogy from the relation between independent variables, number of independent variables and sample size," Balkan medical journal, vol. 30, no. 1, p. 28, 2013.

[37] Y. Rodríguez Aldana, B. Hunyadi, E. Maranon Reyes, V. Rodriguez Rodriguez, and S. Van Huffel, "Nonconvulsive epileptic seizures detection using multiway data analysis," in Proc. of the 25th European Signal Processing Conference, 2017, pp. 1-5.

[38] R. P. Duin, P. Juszczak, P. Paclik, E. Pekalska, D. de Ridder, D. Tax, and S. Verzakov, "Prtools 4-a matlab toolbox for pattern recognition. version 4.1," Delft University of Technology, 2007.

[39] A. Bhattacharyya and R. B. Pachori, "A multivariate approach for patient-specific eeg seizure detection using empirical wavelet transform," IEEE Transactions on Biomedical Engineering, vol. 64, no. 9, pp. 20032015, 2017.

[40] A. Bhattacharyya, R. B. Pachori, A. Upadhyay, and U. R. Acharya, "Tunable-q wavelet transform based multiscale entropy measure for automated classification of epileptic eeg signals," Applied Sciences, vol. 7, no. 4, p. 385, 2017.

[41] T. Fatma, O. Farooq, Y. U. Khan, M. Tripathi, and P. Sharma, "Automatic detection of non-convulsive seizures: A reduced complexity approach," Journal of King Saud University-Computer and Information Sciences, vol. 28 , no. 4 , pp. $407-415,2016$. 